\title{
Analysis of Axial Length in Young Patients with Marfan Syndrome and Bilateral Ectopia Lentis by Z-Scores
}

\author{
Ze-Xu Chen ${ }^{a, b, c, d}$ Jia-Hui Chen ${ }^{a, b, c, d}$ Min Zhang ${ }^{a, b, c, d}$ Tian-Hui Chen ${ }^{a, b, c, d}$ \\ Jia-Lei Zheng ${ }^{a, b, c, d}$ Michael Deng ${ }^{a, b, c, d}$ Ying-Hong Ji ${ }^{a, b, c, d}$ \\ Yong-Xiang Jiang a, b, c, d \\ aEye Institute and Department of Ophthalmology, Eye \& ENT Hospital, Fudan University, Shanghai, China; ${ }^{b} \mathrm{NHC}$ Key \\ Laboratory of Myopia, Fudan University, Shanghai, China; 'Key Laboratory of Myopia, Chinese Academy of Medical

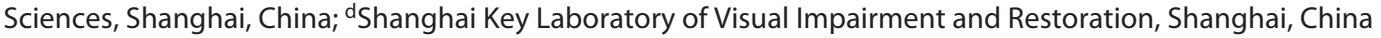

\section{Keywords}

Axial length $\cdot$ Z-score $\cdot$ Marfan syndrome $\cdot$ Ectopia lentis

\begin{abstract}
Introduction: Marfan syndrome (MFS) is characterized by ectopia lentis (EL) and elongated axial length (AL). The characteristics of AL in young patients with MFS and bilateral EL before the lens surgery are not fully understood. Methods: This study reviewed MFS patients under 20 years old with bilateral EL from January 2015 to October 2020. The Z-scores were introduced in terms of the number of standard deviations from the mean of age-matched normative data. Using $Z$-scores, the distribution of $A L$ and influence factors were evaluated. The correlations between AL and other biometrics were analyzed. Results: We reviewed 183 patients and enrolled both eyes. The mean age was $8.44 \pm 4.69$ years. About $36 \%$ of the patients were children under 6 years old. The median AL increased from $23.16 \mathrm{~mm}$ under 5 years old to $26.20 \mathrm{~mm}$ in the 16-20 age group, and when plotted, the trend presented a logarithmic curvature $\left(R^{2}=0.145, p<\right.$ 0.001). The median Z-AL score was 1.24. One-third of eyes
\end{abstract}

Ze-Xu Chen and Jia-Hui Chen contributed equally to this work.

karger@karger.com www.karger.com/ore

Karger"

BOPEN ACCESS
(C) 2021 The Author(s)

Published by S. Karger AG, Basel

This is an Open Access article licensed under the Creative Commons Attribution-NonCommercial-4.0 International License (CC BY-NC) (http://www.karger.com/Services/OpenAccessLicense), applicable to the online version of the article only. Usage and distribution for commercial purposes requires written permission. had Z-score $<0$. About $20 \%$ of the patients had AL difference over $1 \mathrm{~mm}$ between the right and left eyes, and the right one had longer Z-AL scores $(p=0.013)$. The eye complicated with megalocornea $(10,7.04 \%)$ had larger Z-AL scores $(4.72 \pm 3.51$ vs. $1.10 \pm 2.25, p=0.002$ ). A positive correlation was found between $\mathrm{Z}$-AL and Z-corneal curvature radius $(r=0.265, p<0.001)$. Conclusion: Young patients with bilateral EL but small AL should not be excluded from MFS without systematic examination. The age-adjusted Z-score will facilitate further study of the individual variations in $A L$ across different ages.

(c) 2021 The Author(s).

Published by S. Karger AG, Basel

\section{Introduction}

As an important component of the ocular refractive media, a normal crystalline lens is critical to the development of the visual system. Ectopia lentis (EL) refers to the dislocation of the lens from its physiological position, which may cause refractive errors of varying severity, poor visual acuity, and amblyopia in young patients [1]. The major etiology of congenital EL is Marfan syndrome (MFS, OMIM 154700), the prevalence of which ranges

Correspondence to:

Ying-Hong Ji, jiyh_eent@163.com

Yong-Xiang Jiang, yongxiang_jiang@ 163.com 
Fig. 1. Age distribution of MFS patients with bilateral EL. Subdivided groups were marked by different colors: $<5$ years old (yellow), 6-8 years old (gray), 9-11 years old (blue), 12-15 years old (cyan), and 1620 years old (purple). The number of eyes $(n)$ in each group was labeled. EL, ectopia lentis; MFS, Marfan syndrome.

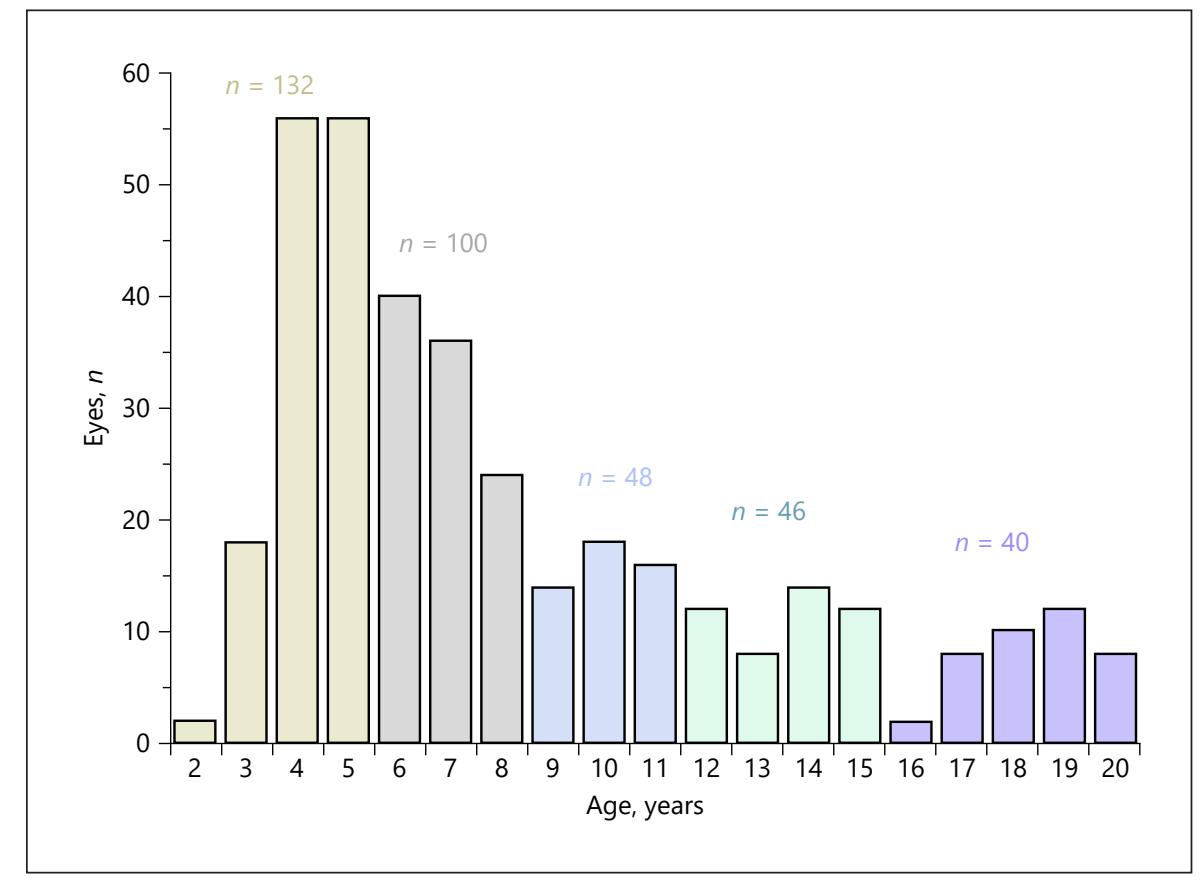

from 4.6 to 6.5 per 100,000 [2-4]. MFS is an autosomal dominant hereditary disease caused by fibrillin-1 (FBN1) gene mutations [5]; fibrillin-1 is the main constituent of the microfilaments, which are the key structural components of the ciliary zonules [6]. Mutations of FBN1 result in the impaired suspension of the crystalline lens in its anatomic position [7]. In addition to EL, the eyes of patients with MFS are characterized by long axial length (AL), low corneal curvatures, severe astigmatism, and dense myopia [8].

Refractive and biometric characteristics of patients with MFS have been reported [9-13]; however, most studies have used a relatively small sample size without control groups. To our knowledge, few investigations have examined the distribution of AL and the correlations between AL and other biometrics before lens surgery in young MFS patients with bilateral EL. In this retrospective study of a relatively large group of patients, we aimed to investigate the overall status of axial development in MFS patients younger than 20 years old and the relationships between AL and patient demographics, including age, sex, family history, and comorbidities. We introduced the age-adjusted Z-scores by using external controls to analyze the correlations of AL development with the corneal curvature radius (CCR), AL/CCR ratio, and white-to-white horizontal corneal diameter (WTW). Our results provide a useful reference for the precise planning of surgical interventions for young MFS patients with bilateral EL.

\section{Methods}

\section{Patient Eligibility}

The study was approved by the Human Research Ethics Committee of the Eye \& ENT Hospital of Fudan University (ChiCTR2000039132) and carried out in accordance with the Declaration of Helsinki. Informed consent in written form was obtained from all patients and the patients' guardians for those under 18 years old. The inclusion criteria were as follows: (1) patients diagnosed as having MFS or possible MFS according to the Ghent-2 nosology [14], (2) patients under 20 years old (because they are defined as "young individuals" in Ghent-2 nosology, who have different diagnostic criteria), and (3) patients with bilateral EL under slit-lamp examination during complete pupillary dilation. Patients with the following features were excluded from the study: (1) lens dislocated into the anterior chamber or vitreous body; (2) a history of traumatic lens dislocation; (3) pre-existing ocular comorbidities, such as congenital cataract, retinal tears, retinal detachment, corneal ectasia, or glaucoma; and (4) the presence of other connective tissue diseases. Both eyes were selected because we found a significant difference in the AL between the 2 eyes of each participant by the paired $t$ test $(p=0.013)$. The recorded patients' ages were the ages at the examination.

\section{Ophthalmic Examinations}

The participants were examined by board-certified ophthalmologists, and their medical and family histories were evaluated. 


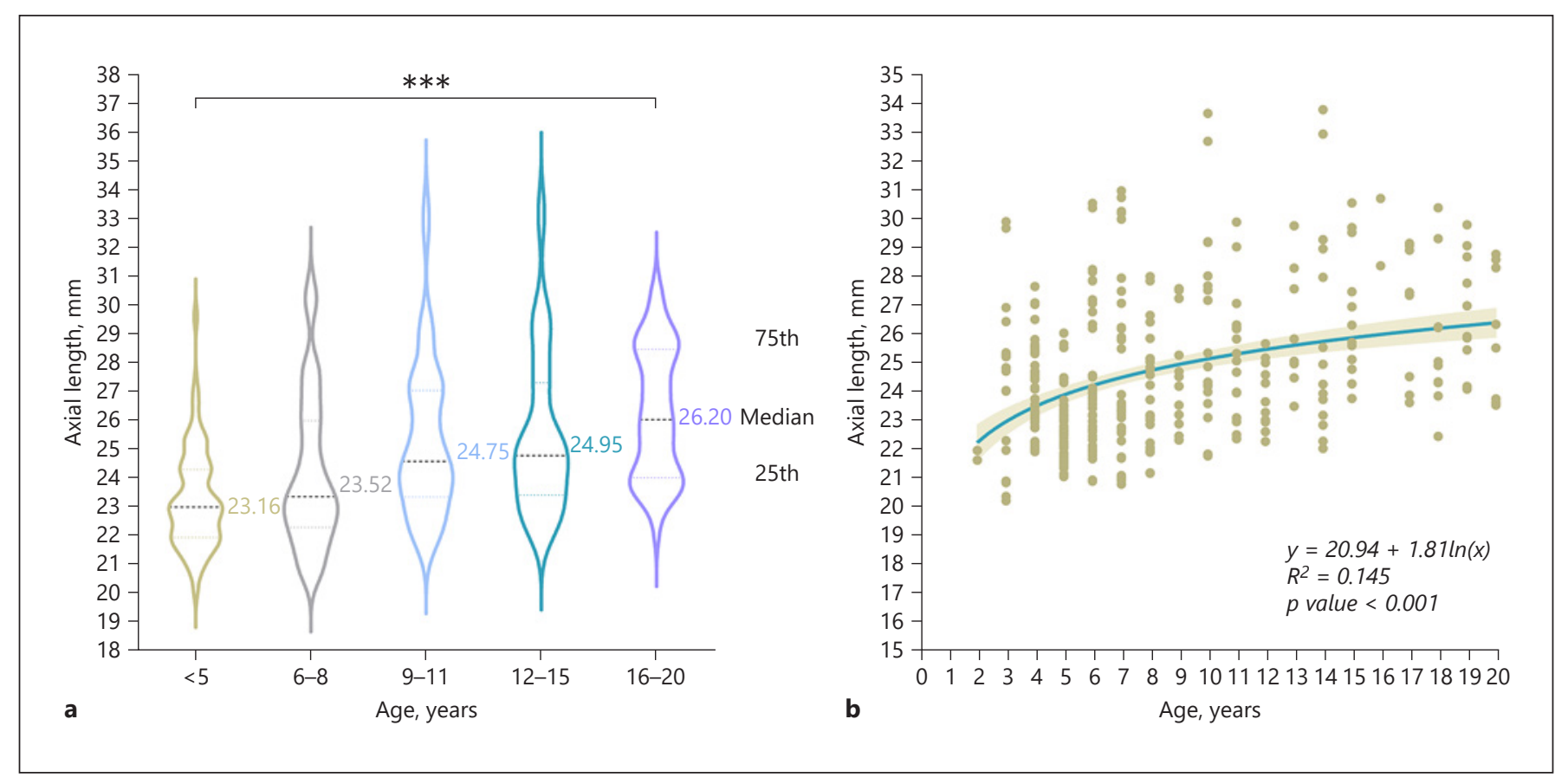

Fig. 2. Differences in AL among MFS patients with bilateral EL of various ages. a The violin graph showed the distribution of AL in different age subgroups. ${ }^{* * *} p<0.001$ (Kruskal-Wallis test). b Scatterplot of AL by patients' ages and fitted logarithmic curve. The shaded area demonstrated the 95\% CI. AL, axial length; EL, ectopia lentis; MFS, Marfan syndrome.

All patients were examined for EL by slit-lamp examination under complete pupillary dilation. Bilateral EL was defined as the displacement of the lens from its normal position in both eyes. The AL, CCR, WTW, and corneal astigmatism were measured using partial coherence interferometry (IOLMaster 500 and 700; Carl Zeiss Meditec AG, Jena, Germany). Five valid AL readings and 3 readings of both CCR and WTW were taken.

\section{Z-Score Calculation}

The AL and other ocular biometrics were compared to agematched normative data published in the literature that were cross-sectional, epidemiological investigations of the Chinese population. The Z-score of AL ( $\mathrm{Z}$-AL) was calculated using the following formula: $\mathrm{Z}-\mathrm{AL}=$ (measured $\mathrm{AL}$ - normative $\mathrm{AL}$ )/normative standard deviation (SD). The Z-score describes the position of a raw value in terms of its distance from the mean when measured in SD units. For example, a Z-score $\geq 2.0$ corresponds to a measurement $\geq 2.0 \mathrm{SD}$ above normal. For patients of $4-18$ years of age, the AL, CCR, and WTW were compared with normative measurement data in the Shandong Children Eye Study [15-17]. For those over 18 years old, the measurements for 18-yearold children in the same study were employed as reference data. For 3-year-old patients, the Shanghai Pudong Eye Study was used as the reference [18] (see online suppl. Table 1; for all online suppl. material, see www.karger.com/doi/10.1159/000517384). Because of a lack of normative measurements for 2 -year-olds in the Chinese population, the Z-score was not analyzed in 1 patient ( 2 eyes). The Z-scores for other biometric parameters, such as Z-CCR and Z-WTW, were calculated similarly.

Axial Length in Young Patients with Marfan Syndrome

\section{Statistical Analysis}

Continuous variables were described in mean \pm SD or median and interquartile range. Categorical variables were described in counts or proportions as appropriate. The distributions for age and ocular biometric parameters were tested for normality with the Kolmogorov-Smirnov test. The one-way analysis of variance or Kruskal-Wallis test was used, as appropriate, to compare the $\mathrm{AL}$ among different age groups. For independent samples, the $t$ test or Wilcoxon test was used to analyze differences in the AL among patients with different factors, including gender, family history, and comorbidities. And, the paired $t$ test or paired Wilcoxon test was used to evaluate the difference in AL between the left and right eye. A logarithmic correlation between AL and age was calculated for MFS patients. Correlations between the Zscore of $\mathrm{AL}$ and $\mathrm{Z}$-scores of other biometric variables were analyzed using Spearman's correlation. All analyses were 2-tailed, and the statistical significance was set as a $p$ value of $<0.05$. Statistical analyses were performed using SPSS version 22.0 (IBM Corp., Armonk, NY, USA).

\section{Results}

\section{Patient Demographics}

The ocular biometric parameters of 183 MFS patients under 20 years old with bilateral EL were collated. Both eyes were enrolled. All participants were Chinese and 


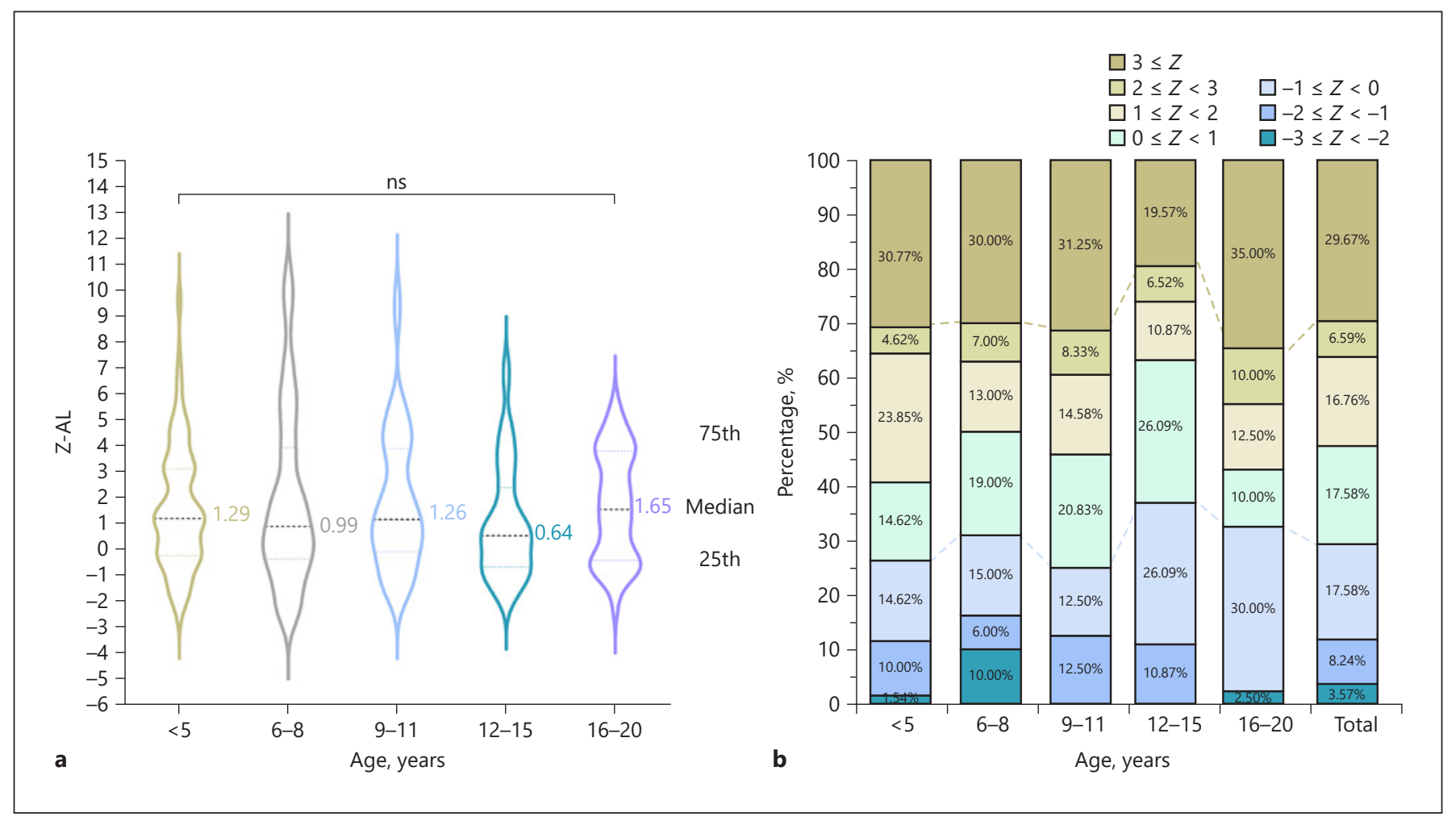

Fig. 3. Differences in the Z-AL score of MFS patients with bilateral EL of different ages. a The violin graph showed the distribution of Z-AL scores in different age subgroups. ns, $p>0.05$ (Kruskal-Wallis test). $\mathbf{b}$ Proportions of $\mathrm{Z}$-AL scores in different age groups. AL, axial length; EL, ectopia lentis; MFS, Marfan syndrome.

$55.49 \%$ were male. The age distribution of the participants is shown in Figure 1, and the mean age was $8.44 \pm$ 4.69 years (range, $2-20$ years). Patients under 6 years old constituted the highest proportion: $36.07 \%$ of the total participants. The number of patients decreased with the advancing age group. Subjects were further stratified by age considering the characteristic of $\mathrm{AL}$ growth in normal children: $\leq 5,6-8,9-11,12-15$, and 16-20 years old.

\section{The AL Distribution}

The AL increased as the patients aged. The distribution of AL did not present a Gaussian distribution $(p<0.001)$. The skewness was 0.970 , and the kurtosis was 0.685 . The overall median AL was $23.97 \mathrm{~mm}\left(p_{25}, 22.71 ; p_{75}, 25.81\right)$. The median $\mathrm{AL}$ and interquartile range in different age groups are shown in Figure 2a. There was a significant difference in the AL among the age subgroups $(p<0.001)$, and the trend in AL increase shown in the scatterplot followed a logarithmic curve $\left(R^{2}=0.145, p<0.001\right)$ (Fig. 2b). Considerable variation in AL was observed among patients of the same age.

\section{The Z-AL Score Distribution}

The Z-AL did not increase as the patients aged. The Z-AL scores did not follow a Gaussian distribution $(p<0.001)$. The skewness was 0.973 , and the kurtosis was 0.255 . The overall median Z-AL was $1.24 \mathrm{~mm}\left(p_{25},-0.22 ; p_{75}, 3.41\right)$. The median AL and interquartile range in different age groups are shown in Figure 3a. The age difference was successfully adjusted by the Z-AL score because there were no statistical differences in the Z-AL score among the different age groups $(p=0.531)$. The distribution of AL within the same age group was well represented by the Z-score (Fig. 3b). The proportion of the Z-AL score shared a similar pattern among the age subgroups. A considerable variation in $\mathrm{Z}$-AL was observed. In all the age groups, about $30 \%$ of patients had a Z-AL score $<0$, and extremely long AL ( $Z$-AL score $\geq 3$ ) was observed in approximately $30 \%$ of patients (Fig. 3b).

\section{Relationship between Z-AL Score with Different Factors}

To further explore the factors influencing the AL of the participants, we analyzed the relationships among AL or Z-AL score and laterality, sex, and family history (Table 1). 
Table 1. Comparison of the AL and Z-AL between different factors according to age groups

\begin{tabular}{lccc}
\hline \multirow{2}{*}{ Factor } & \multicolumn{2}{l}{ Z-AL score } & \\
\cline { 2 - 4 } & eyes, $n$ & mean \pm SD & $p$ value \\
\hline Laterality & 182 & $1.85 \pm 2.72$ & \\
Right & 182 & $1.62 \pm 2.64$ & $\mathbf{0 . 0 1 3}$ \\
Left & & & \\
\hline Sex & 202 & $1.86 \pm 2.76$ & 0.267 \\
Male & 162 & $1.59 \pm 2.58$ & \\
Female & & & \\
\hline Family history (MFS) & 144 & $1.70 \pm 2.56$ & 0.907 \\
Positive & 220 & $1.77 \pm 2.76$ & \\
Negative & & & \\
\hline Microspherophakia & 20 & $2.53 \pm 2.64$ & 0.145 \\
Positive & 346 & $1.69 \pm 2.68$ & \\
Negative & & & \\
\hline Megalocornea & 10 & $4.72 \pm 3.51$ & $\mathbf{0 . 0 0 2}$ \\
Positive & 132 & $1.10 \pm 2.25$ & \\
Negative &
\end{tabular}

$p$ values $<0.05$ are highlighted in bold (laterality, paired Wilcoxon test) (gender, family history, and comorbidities, Wilcoxon test). AL, axial length; MFS, Marfan syndrome; SD, standard deviation.

The right eyes had slightly but significantly larger Z-AL scores than the left ones $(1.85 \pm 2.72$ vs. $1.62 \pm 2.64, p=$ $0.013)$. The scatterplot showed a considerable axial difference ( $\triangle \mathrm{AL}=\mathrm{AL}$ of the right eye $-\mathrm{AL}$ of the left eye) between the right and left eyes (Fig. 4a). About one-fifth of patients had the absolute value of $\Delta \mathrm{AL}$ over $1 \mathrm{~mm}$, and a longer $\mathrm{AL}$ in the right eye was observed in 101 patients (55.19\%) (Fig. 4b). Males seemed to have longer AL, but the difference was not significant $(p=0.267)$. There was no difference between patients with or without a family history of MFS ( $p=0.907)$. In the 20 eyes $(10.87 \%)$ with microspherophakia, the AL appeared to be larger, but the difference was not statistically significant $(p=0.145)$. Of the 142 eyes (38.80\%) with qualified WTW measurements, megalocornea (WTW $\geq 13$ ) was found in $10(7.04 \%)$. MFS eyes complicated by megalocornea had larger Z-AL scores $(4.72 \pm 3.51$ vs. $1.10 \pm 2.25, p=0.002)$.

\section{Correlation between AL with Biometry Parameters as Z-Scores}

Z-AL scores were plotted against Z-CCR, Z-WTW, AL/ CCR ratio, and corneal astigmatism. There was a positive correlation between Z-AL and Z-CCR $(r=0.265, p<0.001)$

Axial Length in Young Patients with

Marfan Syndrome
(Fig. 5a) and a positive correlation between Z-AL and AL/ CCR ratio $(r=0.762, p<0.001)$ (Fig. 5b). No correlation was found between Z-AL and Z-WTW $(r=0.052, p=0.537)$ (Fig. 5c). A mild but significant correlation was observed between Z-AL and AST ( $r=0.151, p=0.004)$ (Fig. 5d).

\section{Discussion}

About $60 \%$ of patients with MFS have EL, which is usually bilateral, progressive, and has a superior direction [8]. The implantation of an intraocular lens (IOL) plays a crucial part in the postoperative visual rehabilitation of children. The AL is the most important factor affecting the accuracy of IOL power, and a 1-mm error of measurement can induce an approximate $2.5-\mathrm{D}$ refractive error [19]. Previous investigations have shown the $\mathrm{AL}$ in the eyes of patients with EL is different from that in normal eyes [20]. However, there have been few studies of the characteristics of the AL in young MFS patients before lens surgery. In this study, we recruited 183 MFS patients with bilateral EL to investigate the distribution and influence factors of AL in the developing age. The age-adjusted $\mathrm{Z}$-score is a powerful tool to study the influencing factors and the correlation between $\mathrm{AL}$ and other biometrics.

Here, we present the distribution of AL in MFS patients with bilateral EL at a relatively young age. Similar to the results for the normative cohort [21], there was a positive correlation between $\mathrm{AL}$ and age in the current study. A logarithmic correlation between AL and age was found in congenital cataract patients [22]. AL increases dramatically in the first 2 years of life and then grows at a slower rate until adulthood [22]. The AL growth trend for patients in the current study also presented a logarithmic curvature. However, because of the limited number of patients under 3 years old, the high growth rate normally seen in the early stage of the logarithmic curve is absent in this study. Previous studies found that EL can manifest at an early age: EL is recognized at age 0 in $38 \%$ of patients with neonatal MFS [23], and $12.5 \%$ of MFS patients show EL before 3 years of age [8]. The prevalence might have been underestimated because of the difficulties for young patients expressing themselves and poor cooperation under ophthalmic examination. The distribution of $\mathrm{AL}$ among MFS patients under 3 years old is uncertain and warrants further study.

The Z-score has been used by cardiologists to adjust for body surface area when measuring aortic sinus diameters in various individuals [24], and new Z-scores have 


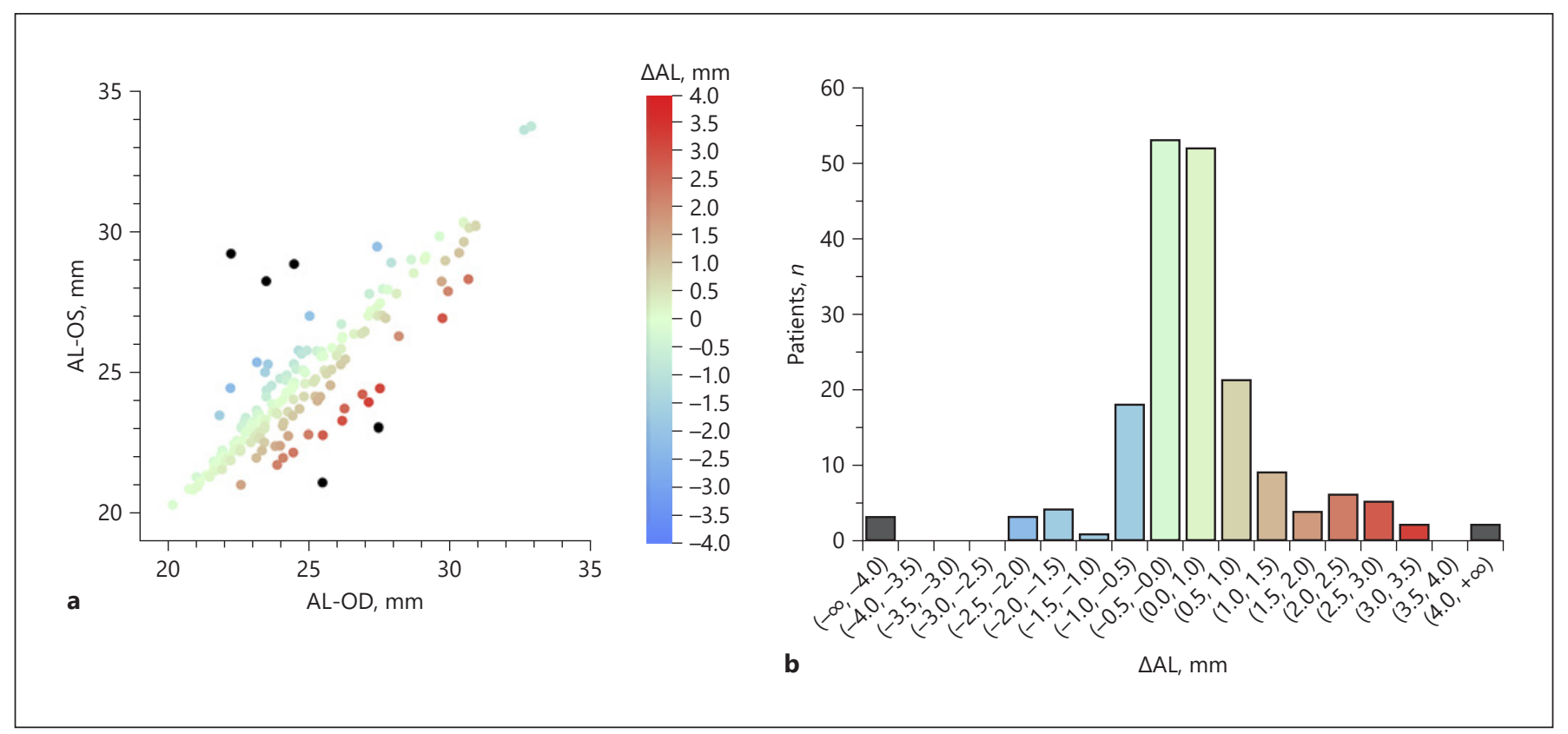

Fig. 4. Analysis of AL difference of the right and left eyes of MFS patients with bilateral EL. a Scatterplot of AL in the right $(\mathrm{AL}-\mathrm{OD})$ and left $(\mathrm{AL}-\mathrm{OS})$ eyes. The difference of $\mathrm{AL}(\triangle \mathrm{AL})$ was manifested in different colors. $\triangle \mathrm{AL}$ over $4.0 \mathrm{~mm}$ or below $-4.0 \mathrm{~mm}$ was marked in dark. $\mathbf{b}$ Distribution of the difference of $\mathrm{AL}(\triangle \mathrm{AL}=\mathrm{AL}-\mathrm{OD}-\mathrm{AL}-$ OS). AL, axial length; EL, ectopia lentis; MFS, Marfan syndrome.

been developed to normalize age, sex, height, and weight [25]. A Z-score $\geq 2.0$ ( $\geq 3.0$ for patients under 20 years old) is a major criterion in the diagnosis of MFS [14]. However, the age-adjusted Z-score introduced in the current study was not intended to assist in making a diagnosis based on ocular biometrics, which has been excluded from the 2010 Ghent-2 criteria due to uncertain specificity [14]. In this study, the Z-scores successfully normalized the age differences in $\mathrm{AL}$ and other parameters by comparing age-matched published normative values.

The distribution of $\mathrm{AL}$ across the age groups was well represented by the $\mathrm{Z}$-score. The median $\mathrm{AL}$ was $1.24 \mathrm{SD}$ from the normative mean measurement. About $30 \%$ of eyes had relatively long AL (Z-AL score $\geq 3$ ) and the largest had a Z-score of 10.68. A long AL has been proven to be a risk factor for retinal detachment [26] and open-angle glaucoma [27] in the eyes of MFS patients. Thus, patients with longer AL should have more intensive ophthalmic exams, especially when they have a visual complaint. Although a long AL has been recognized as an ocular manifestation of MFS, our study showed that about $30 \%$ of the eyes had a short AL (Z-AL score $<0$ ), and the proportion was similar among the different age groups. Thus, young patients with bilateral EL but small
AL should not be simply diagnosed as having idiopathic EL without considering the systematic features. Since the manifestations of MFS can be atypical at a young age [28], a genetic test or careful follow-up is strongly recommended.

Considerable variation was observed among those of the same age, indicating that the development of the AL in MFS patients is associated with multiple factors in addition to age. However, the sex and family history of MFS were not associated with differences in AL. One possible explanation for the AL variations is the allelic heterogeneity of FBN1 mutations. More than 3,000 mutations of FBN1 are known today [29], but only $14.20 \%$ of the mutations are recurrent [30]. The misfolded fibrillin-1 and the disorganized elastin in the scleral stroma could lead to stretching of the sclera through the 2 physiological functions of fibrillin-1 (structure component or TGF- $\beta$ signaling mediator) and by different underlying genetic mechanisms (haploinsufficiency or dominant-negative effect) [30]. In addition, the heterogeneity of FBN1 mutations could lead to various degrees of EL, which accelerates globe growth via defocus or deprivation. Further genotypic-phenotypic analyses are required to understand the variations in AL and severity of EL. The Z-score will be 


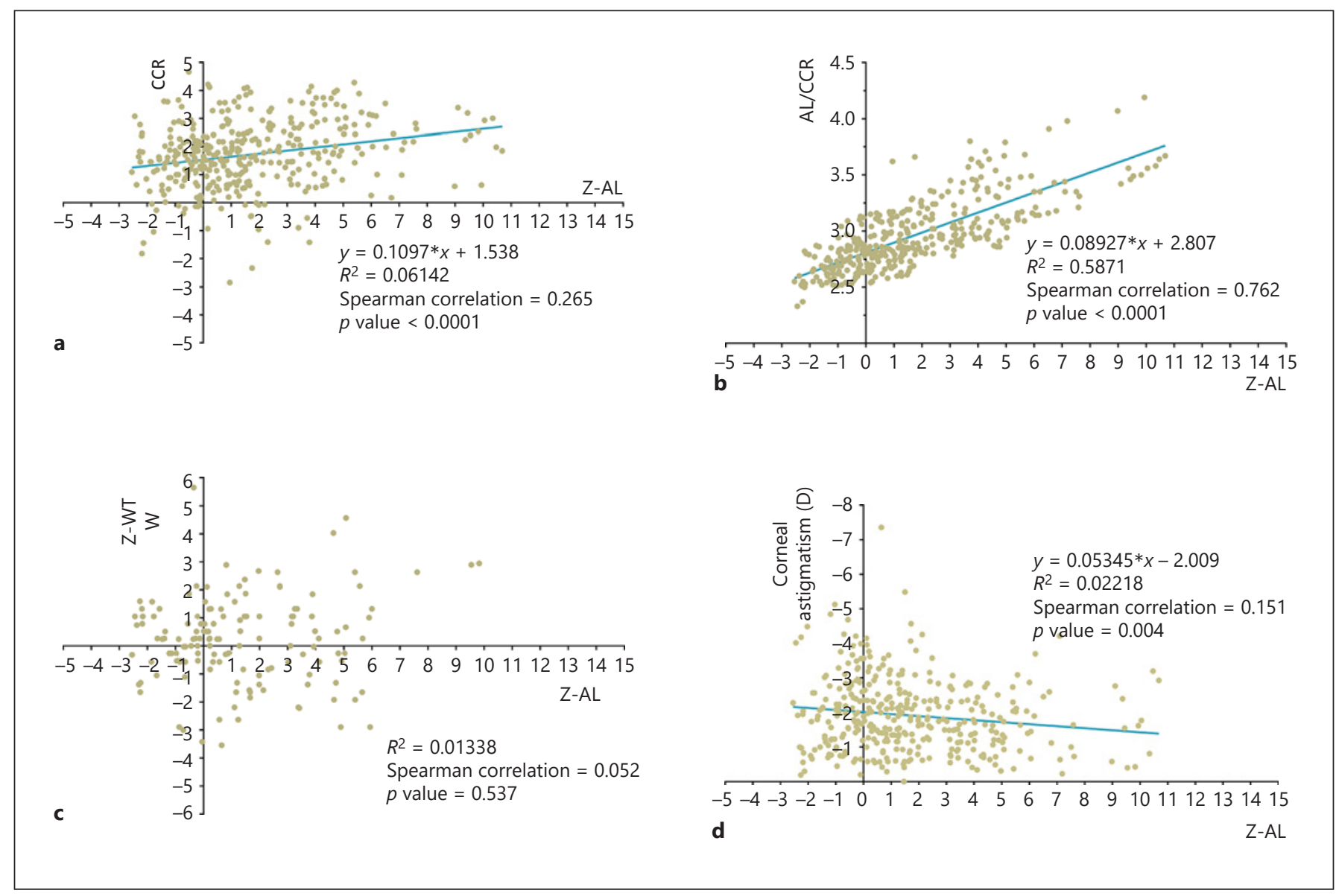

Fig. 5. Scatterplots showing correlations (Spearman's correlation) between the Z-AL score and Z-CCR score (a), AL/ CCR ratio (b), Z-WTW score (c), and corneal astigmatism (d) in MFS patients with bilateral EL. AL, axial length; CCR, corneal curvature radius; MFS, Marfan syndrome; WTW, white-to-white horizontal corneal diameter; EL, ectopia lentis.

helpful when analyzing FBN1 mutations and ocular phenotypes in young patients of different ages. However, a considerable AL difference is observed in bilateral eyes of the same patient. About $20 \%$ of patients had AL differences over $1 \mathrm{~mm}$ between the bilateral eyes, and the greatest difference is $7 \mathrm{~mm}$, indicating that environmental factors and epigenetic factors may contribute to the difference. The aniso-axial length can lead to anisometropia and challenge the choice of IOL power. The AL of the right eyes was significantly longer than the left ones, probably because of dextromanuality and right dominance of eyes [31]. However, further studies are warranted to test this hypothesis.

A previous study described the ocular biometrics of MFS eyes based on age stratification [32]. Although AL is an important indicator of eyeball development, the relationships between $\mathrm{AL}$ and other biometric parameters have been scarcely reported in MFS patients. Correlations among AL and other biometrics were analyzed in the form of the age-adjusted Z-scores, and a positive correlation was found between AL and CCR. The decreasing power of the cornea appears to play an emmetropizing role in response to increasing AL; however, this compensatory capacity reportedly reaches its limit at an AL/ CCR ratio of 3 [33]. In the linear regression model used in the current study, the AL/CCR ratio exceeded 3 when the Z-AL score was above 2.16. However, the correlation between AL and preoperative spherical equivalent was not studied because the displaced and deformed lens can affect the total refractive power. Nevertheless, we hypothesized that in the postoperative eyes with centered and stabilized IOL positions, a long-term myopic shift is 
expected in the developing eyes when the Z-AL exceeds 2.16. Therefore, the selection of IOL power deserves more careful consideration in those patients to achieve the refractive target. Microspherophakia and megalocornea are sometimes found in eyes of MFS patients [34, 35]. To the best of our knowledge, the prevalence of these 2 comorbidities in MFS patients with bilateral EL has never been reported. Our study indicated that approximately $10 \%$ of eyes of MFS patients were complicated by microspherophakia, the AL of which seemed to be greater than that of normal eyes but not to a statistically significant degree. Megalocornea was found in around 7\% of MFS eyes. Although WTW measurements did not change proportionally with AL growth, MFS eyes complicated by megalocornea had significantly larger AL on average. Previous studies have shown that there was higher corneal astigmatism in the eyes of MFS patients $[36,37]$. Additionally, we found that patients with smaller AL generally had higher corneal astigmatism. Thus, residual astigmatism from the cornea after lens aspiration and IOL implantation, especially in MFS eyes with smaller AL, is a challenge for postoperative visual rehabilitation.

The results of this study must be assessed within the context of its limitations. First, the data are not a complete representation of MFS patients and only relate to surgical patients with bilateral EL, which prevented us from detecting differences in eyes with unilateral EL or without EL. Second, the external controls used in our study were school-based, cross-sectional studies of young Chinese individuals from a large sample size. The conclusions should be viewed with caution for other ethnic populations. Third, adult patients were excluded from the current study, as AL was shown to be stable in adults $(>30$ years old) with MFS during a 10-year follow-up [38]. The cross-sectional nature of our study also limited our ability to track changes in AL data over time. Despite these limitations, the results of this study describe the overall distribution of AL in the eyes of MFS patients with bilateral EL and cover a relatively young patient group compared to the available literature.

\section{Conclusion}

The mean AL of MFS was found to be longer than the age-matched controls, especially in the eyes complicated by megalocornea. But one-third of patients still had an AL below the normative average. The considerable individual variation indicates that young patients with bilateral EL but small AL should not be excluded from MFS diagnoses without systematic examinations. The Z-scores normalized the age difference in AL and other parameters by comparing age-matched published normative values, and this technique facilitates the further study of the influence factors of AL in young patients of different ages. A full understanding of the AL will be helpful for surgical planning and complication monitoring.

\section{Statement of Ethics}

The study was approved by the Human Research Ethics Committee of the Eye \& ENT Hospital of Fudan University (ChiCTR2000039132) and carried out in accordance with the Declaration of Helsinki. Informed consent in written form was obtained from all patients and the patients' guardians for those under 18 years old.

\section{Conflict of Interest Statement}

The authors have no conflicts of interest to declare.

\section{Funding Sources}

This study was supported by the National Natural Science Foundation of China (Grant No. 81770908), the National Natural Science Foundation of China (Grant No. 82070943), the Shanghai Science and Technology Commission (Scientific Innovation Action Plan, Grant No. 18411965200), and the Shanghai Science and Technology Commission (Scientific Innovation Action Plan, Grant No. 20Y11911000). The sponsors played no role in the study design, data collection, data analysis, manuscript preparation, nor the decision to submit the manuscript for publication.

\section{Author Contributions}

Z.-X.C. and J.-H.C. were involved in study design, data collection, and manuscript preparation. M.Z. and J.-L.Z. participated in data acquisition. M.D. and T.-H.C critically reviewed the manuscript. Y.-H.J. and Y.-X.J. supervised the project. All the authors have read and approved the final manuscript.

\section{Data Availability Statement}

The datasets generated and/or analyzed during the present study are not publicly available (obtained from Eye \& ENT Hospital, of Fudan University, Shanghai repository), but are available from the corresponding author upon reasonable request. 


\section{References}

1 Nemet AY, Assia EI, Apple DJ, Barequet IS. Current concepts of ocular manifestations in marfan syndrome. Surv Ophthalmol. 2006; 51(6):561-75.

2 Groth KA, Hove H, Kyhl K, Folkestad L, Gaustadnes M, Vejlstrup N, et al. Prevalence, incidence, and age at diagnosis in marfan syndrome. Orphanet J Rare Dis. 2015;10:153.

3 Fuchs J. Marfan syndrome and other systemic disorders with congenital ectopia lentis. A danish national survey. Acta Paediatr. 1997; 86(9):947-52.

4 De Paepe A, Devereux RB, Dietz HC, Hennekam RC, Pyeritz RE. Revised diagnostic criteria for the marfan syndrome. Am J Med Genet. 1996;62(4):417-26.

5 Kainulainen K, Pulkkinen L, Savolainen A, Kaitila I, Peltonen L. Location on chromosome 15 of the gene defect causing marfan syndrome. N Engl J Med. 1990;323(14):935-

6 De Maria A, Wilmarth PA, David LL, Bassnett S. Proteomic analysis of the bovine and human ciliary zonule. Invest Ophthalmol Vis Sci. 2017;58(1):573-85

7 Jones W, Rodriguez J, Bassnett S. Targeted deletion of fibrillin-1 in the mouse eye results in ectopia lentis and other ocular phenotypes associated with marfan syndrome. Dis Model Mech. 2019;12(1):dmm037283.

8 Maumenee IH. The eye in the marfan syndrome. Trans Am Ophthalmol Soc. 1981;79: 684-733.

9 Salchow DJ, Gehle P. Ocular manifestations of marfan syndrome in children and adolescents. Eur J Ophthalmol. 2019;29(1):38.

10 Gehle P, Goergen B, Pilger D, Ruokonen P, Robinson PN, Salchow DJ. Biometric and structural ocular manifestations of marfan syndrome. PLoS One. 2017;12(9):e0183370.

11 Drolsum L, Rand-Hendriksen S, Paus B, Geiran OR, Semb SO. Ocular findings in 87 adults with ghent-1 verified marfan syndrome. Acta Ophthalmol. 2015;93(1):46-53.

12 Konradsen TR, Koivula A, Kugelberg M, Zetterström C. Corneal curvature, pachymetry, and endothelial cell density in marfan syndrome. Acta Ophthalmol. 2012;90(4): 375-9.

13 Heur M, Costin B, Crowe S, Grimm RA, Moran R, Svensson LG, et al. The value of keratometry and central corneal thickness measurements in the clinical diagnosis of marfan syndrome. Am J Ophthalmol. 2008 145(6):997-1001.

14 Loeys BL, Dietz HC, Braverman AC, Callewaert BL, De Backer J, Devereux RB, et al. The revised ghent nosology for the marfan syndrome. J Med Genet. 2010;47(7):476-85.
15 Zhang YY, Jiang WJ, Teng ZE, Wu JF, Hu YY, $\mathrm{Lu}$ TL, et al. Corneal curvature radius and associated factors in chinese children: the shandong children eye study. PLoS One. 2015; 10(2):e0117481.

16 Lu TL, Wu JF, Ye X, Hu YY, Wu H, Sun W, et al. Axial length and associated factors in children: the shandong children eye study. Ophthalmologica. 2016;235(2):78-86.

17 Jiang WJ, Wu H, Wu JF, Hu YY, Lu TL, Sun $\mathrm{W}$, et al. Corneal diameter and associated parameters in chinese children: the shandong children eye study. Clin Exp Ophthalmol. 2017;45(2):112-9.

18 Zhao KK, Yang Y, Wang H, Li L, Wang ZY, Jiang $\mathrm{F}$, et al. Axial length/corneal radius of curvature ratio and refractive development evaluation in 3- to 4-year-old children: the shanghai pudong eye study. Int J Ophthalmol. 2019;12(6):1021-6.

19 Wilson ME, Trivedi RH. Axial length measurement techniques in pediatric eyes with cataract. Saudi J Ophthalmol. 2012;26(1):137

20 Zhang Y, Jin G, Cao Q, Lin J, Lin J, Wang Y, et al. Distribution of axial length in chinese congenital ectopia lentis patients: a retrospective study. BMC Ophthalmol. 2017;17(1):113.

21 Tideman JWL, Polling JR, Vingerling JR, Jaddoe VWV, Williams C, Guggenheim JA, et al. Axial length growth and the risk of developing myopia in european children. Acta Ophthalmol. 2018;96(3):301-9.

22 Lin H, Lin D, Chen J, Luo L, Lin Z, Wu X, et al. Distribution of axial length before cataract surgery in chinese pediatric patients. Sci Rep. 2016;6(1):23862.

23 Faivre L, Masurel-Paulet A, Collod-Béroud G, Callewaert BL, Child AH, Stheneur C, et al. Clinical and molecular study of 320 children with marfan syndrome and related type i fibrillinopathies in a series of 1009 probands with pathogenic fbn 1 mutations. Pediatrics. 2009;123(1):391-8.

24 Roman MJ, Devereux RB, Kramer-Fox R, O'Loughlin J. Two-dimensional echocardiographic aortic root dimensions in normal children and adults. Am J Cardiol. 1989;64(8): 507-12.

25 Devereux RB, de Simone G, Arnett DK, Best LG, Boerwinkle E, Howard BV, et al. Normal limits in relation to age, body size and gender of two-dimensional echocardiographic aortic root dimensions in persons $\geq 15$ years of age. Am J Cardiol. 2012;110(8):1189-94.

26 Fan F, Luo Y, Liu X, Lu Y, Zheng T. Risk factors for postoperative complications in lensectomy-vitrectomy with or without intraocular lens placement in ectopia lentis associated with Marfan syndrome. Br J Ophthalmol. 2014;98(10):1338-42.

27 Xu W, Kurup SP, Fawzi AA, Durbin MK, Maumenee IH, Mets MB. Comparative data on sd-oct for the retinal nerve fiber layer and retinal macular thickness in a large cohort with marfan syndrome. Ophthalmic Genet. 2017;38(1):34-8.

28 Chandra A, Patel D, Aragon-Martin JA, Pinard A, Collod-Béroud G, Comeglio P, et al. The revised ghent nosology; Reclassifying isolated ectopia lentis. Clin Genet. 2015;87(3):284-7.

29 Sakai LY, Keene DR. Fibrillin protein pleiotropy: acromelic dysplasias. Matrix Biol. 2019; 80:6-13.

30 Faivre L, Collod-Beroud G, Loeys BL, Child A, Binquet C, Gautier E, et al. Effect of mutation type and location on clinical outcome in 1,013 probands with marfan syndrome or related phenotypes and fbn 1 mutations: an international study. Am J Hum Genet. 2007; 81(3):454-66.

31 Jiang S, Chen Z, Bi H, Xia R, Shen T, Zhou L, et al. Elucidation of the more myopic eye in anisometropia: the interplay of laterality, ocular dominance, and anisometropic magnitude. Sci Rep. 2019;9(1):9598.

32 Chen J, Jing Q, Tang Y, Qian D, Lu Y, Jiang Y. Age differences in axial length, corneal curvature, and corneal astigmatism in marfan syndrome with ectopia lentis. J Ophthalmol. 2018;2018:1-8.

33 González Blanco F, Sanz Ferńandez JC, Muńoz Sanz MA. Axial length, corneal radius, and age of myopia onset. Optom Vis Sci. 2008;85(2):89-96.

34 Farat JG, Queiroz MFN, Lottelli AC. Bilateral clear lens extraction and intraocular lens implantation in a child with microspherophakia and marfan syndrome. Arq Bras Oftalmol. 2019;82(1):62-4.

35 Consul BN, Mehrotra AS, Mathur GB. Marfan's syndrome with bilateral megalocornea and subluxated cataractous lenses. J All India Ophthalmol Soc. 1966;14(6):262-3.

36 Zhang Y, Jin G, Young CA, Cao Q, Lin J, Lin $\mathrm{J}$, et al. Analysis of corneal astigmatism before surgery in chinese congenital ectopia lentis patients. Curr Eye Res. 2018;43(8):972-6.

37 Chen J, Jing Q, Tang Y, Qian D, Lu Y, Jiang Y. Corneal curvature, astigmatism, and aberrations in marfan syndrome with lens subluxation: evaluation by pentacam hr system. Sci Rep. 2018;8(1):4079.

38 Sandvik GF, Vanem TT, Rand-Hendriksen S, Cholidis S, Saethre M, Drolsum L. Ten-year reinvestigation of ocular manifestations in marfan syndrome. Clin Exp Ophthalmol. 2019;47(2):212-8. 